\title{
MUC1 and MUC5AC Acting on Helicobacter pylori-Related Deficiency and Solid Syndrome of Spleen and Stomach
}

\author{
Ling Hu (D), ${ }^{1}$ Wanqun Chen, ${ }^{2}$ Ming Cheng, ${ }^{1}$ Ting Zhang, ${ }^{1}$ Shaoyang Lan, \\ Peiwu Li, ${ }^{3}$ and Weijing Chen ${ }^{1}$ \\ ${ }^{1}$ Institute of Gastroenterology, Guangzhou University of Chinese Medicine, Guangzhou 510405, China \\ ${ }^{2}$ Chongqing Hospital of Traditional Chinese Medicine, Chongqing 400037, China \\ ${ }^{3}$ The First Affiliated Hospital, Guangzhou University of Chinese Medicine, Guangzhou 510405, China \\ Correspondence should be addressed to Ling Hu; hl.cn@139.com
}

Received 8 February 2018; Accepted 14 March 2018; Published 23 April 2018

Academic Editor: Victor Kuete

Copyright (C) 2018 Ling Hu et al. This is an open access article distributed under the Creative Commons Attribution License, which permits unrestricted use, distribution, and reproduction in any medium, provided the original work is properly cited.

\begin{abstract}
To investigate the relationship of MUC1, MUC5AC, and the syndrome of spleen and stomach, 109 subjects (34 peptic ulcer (PU), 62 chronic gastritis (CG), and 13 healthy volunteers (CON)) were included. All the subjects included were surveyed with questionnaire to classify them into damp-heat syndrome of spleen and stomach (DHSS), spleen-qi deficiency syndrome (SQD), and CON, examined by gastric endoscope, and biopsied. Rapid urease and methylene blue staining (MBS) were performed on every subject to diagnose for Helicobacter pylori (Hp) infection, and both were defined as Hp-positive. Hematoxylin and eosin (HE) staining was performed on every specimen to explore the histomorphology, inflammatory degree, and inflammatory activity of different groups; then Elivision ${ }^{\mathrm{TM}}$ plus kit was used to test the expression of MUC1 and MUC5AC. All the results of digital images were reviewed by two experts blindly. The inflammatory degree with $\mathrm{Hp}$ infection was higher than those uninfected or CON, but no significant difference was found between DHSS and SQD. And the expressions of MUC5AC with positive Hp was higher than those with negative $\mathrm{Hp}$ or CON regardless of the deficiency and solid syndrome of spleen-stomach but not for MUC1. We speculate that the deficiency and solid syndrome of spleen-stomach is a condition like Tai Ji symbol of dynamic equilibrium, showing the higher expression of MUC5AC but no change of MUC1 in the circumstance of Hp infection.
\end{abstract}

\section{Introduction}

It is well established that Helicobacter pylori $(\mathrm{Hp})$ is the main etiologic factor in a range of pathologies including chronic gastritis (CG), peptic ulcer (PU), and even gastric cancer (GC) $[1,2]$. The theory that intestine GC is a multistep process starting with CG and progressing through atrophy, intestinal metaplasia (IM), and dysplasia triggered by $\mathrm{Hp}$ is well known [3]. Therefore, the multiple and complicated disruptions of organism caused by $\mathrm{Hp}$ have long been the research highlights.

Previous studies have shown that infection with $\mathrm{Hp}$ is able to induce a cascade of innate and adaptive immune response for the gastric mucosa [4, 5]; furthermore, triggered by $\mathrm{Hp}$ infection, the alteration of mucins (MUCs) in the gastric epithelium and their functions have been widely investigated [6-9]. In particular, MUC1 and MUC5AC are believed to be the most critical proteins for protection from $\mathrm{Hp}$ or in the process of carcinogenesis, which have previously been assumed. On one hand, based on the in vitro and mouse model research, studies have elucidated that Hp dwelling exerts the reduction of MUC1 expression due to the mucosal barrier injury [9-11], whereas, on the other hand, it was suggested that glycan-rich niche produced by mucins provides a preferential binding point for Hp [9]; notably, the abnormal expression of MUCl was recognized as oncogene in the development of gastric carcinomas $[12,13]$. In contrast, the expression of MUC5AC was proven to be reduced in the gastric endoscopic biopsy specimens with Hp infection [14], and the significant decrease was demonstrated to represent a marker of worse survival probability in GC [15]. In conclusion, as the critical mucin and the major receptor for $\mathrm{Hp}$, the dual role of MUC1 and MUC5AC can be considered as powerful twoedged sword. 
TABLE 1: Clinical parameters for all included individuals.

\begin{tabular}{|c|c|c|c|c|c|c|}
\hline \multirow{2}{*}{ Group } & \multirow{2}{*}{ Number } & \multicolumn{2}{|c|}{ Gender } & \multicolumn{2}{|c|}{ Diseases } & \multirow{2}{*}{$\begin{array}{c}\text { Age (Y) } \\
\bar{\chi} \pm S\end{array}$} \\
\hline & & M & FEM & PU & CG & \\
\hline DHSS & 68 & 40 & 28 & 32 & 36 & $39.84 \pm 10.44$ \\
\hline $\mathrm{Hp}(+)$ & 39 & 19 & 20 & 16 & 23 & $40.62 \pm 10.76$ \\
\hline $\mathrm{Hp}(-)$ & 29 & 21 & 8 & 6 & 23 & $38.79 \pm 10.10$ \\
\hline SQD & 28 & 15 & 13 & 2 & 26 & $41.75 \pm 9.24$ \\
\hline $\mathrm{Hp}(+)$ & 13 & 1 & 12 & 1 & 12 & $41.84 \pm 8.60$ \\
\hline $\mathrm{Hp}(-)$ & 15 & 2 & 13 & 1 & 14 & $41.66 \pm 10.06$ \\
\hline $\mathrm{CON}$ & 14 & 8 & 6 & - & - & $38.75 \pm 8.64$ \\
\hline
\end{tabular}

Notes. There is no statistical difference for the gender and age among the groups of DHSS, SQD, and CON $(P>0.05)$. Hp $(+)$ : Helicobacter pylori (Hp) positive; $\mathrm{Hp}(-)$ : Hp negative; SQD: spleen-qi deficiency syndrome; CON: control group; M: male; FEM; female; PU: peptic ulcer; CG: chronic gastritis; -: none.

On the basis of holism concept and syndrome differentiation, Chinese medicine (CM) has been paid more attention recently [16, 17]. And syndrome or Zheng differentiation is the critical step in clinic; thus our team has long been contributing to the research of solid and deficiency syndrome of spleen and stomach. The establishment of the animal model of damp-heat syndrome of spleen and stomach (DHSS) and the diagnostic standards of spleen-qi deficiency syndrome (SQD) laid a solid foundation for the research on the relationship of inflammatory cytokines and syndromes triggered by Hp [18-21]. Therefore, based on the previous studies, we hypothesize that, in the circumstance of $\mathrm{Hp}$ infection, MUC1 and MUC5AC may be involved in solid or deficiency syndrome of spleen and stomach.

\section{Materials and Methods}

The present study was approved by the ethics committee of the First Affiliated Hospital of Guangzhou University of Chinese Medicine, and each individual gave signed informed consent.

2.1. Materials and Chemical Regents. The gastric endoscope was obtained from Olympus (Nagano, Japan), and the rapid urease was obtained from Kedi (Guangzhou, China). Monoclonal antibody of MUC1 and MUC5AC was purchased from ZSGB-BIO (Beijing, China), and the cytokine assay of Elivision plus kit was obtained from Maxin (Fujian, China). The microscope used in this study was Olympus (Nagano, Japan).

2.2. Subjects Selection. From March 2010 to March 2011, 109 pairs of gastric endoscopic biopsy specimens, including 34 PU, 62 CG, and 13 healthy volunteers, were collected from the First Affiliated Hospital of Guangzhou University of Chinese Medicine. The diagnostic of CG and PU was reference to the consensus of CG in China and diagnostic criteria of Lancet, respectively $[22,23]$. The diagnostic standards of Hp infection were followed by the associated detection technique [24-26]. Moreover, by reference to the previous study of our team and the state administration of CM in 2002, the diagnostics of DHSS and SQD were established [21, 27]. Similarly, the inclusion and exclusion criteria were the same as our previous research $[21,28]$. And the detailed information of all subjects included in this study is described in Table 1.

In order to evaluate the symptoms and sighs, all the subjects included in the present research were surveyed by two experts of our team in a scientific, objective, and professional way.

2.3. Sample Preparation. All the subjects included were asked to be examined by the gastric endoscopy, and two samples (a pair) were collected from each stomach antrum, namely, from the greater curvature and the opposite position, respectively. In order to make the initial diagnosis of $\mathrm{Hp}$ infection, one of the samples was tested with a rapid urease immediately; simultaneously, the other was fixed with formalin. Aiming to test $\mathrm{Hp}$ infection in a histological way, the specimens were paraffin-embedded and sectioned, and methylene blue staining (MBS) was performed. Only those with double positive results of a rapid urease and MBS were defined as Hp positive.

In order to observe the morphological characteristic of each specimen, hematoxylin and eosin (HE) staining was prepared routinely. In accordance with the consensus of 2006 $[23,29]$, two professional experts examine the degree of inflammation, inflammatory activity, and Hp infection condition as none, mild, moderate, and severe independently and blindly.

2.4. Mucins Protein Assay. Following the Elivision plus kit manufacturer's instruction, the expression of MUC1 and MUC5AC was detected by immunohistochemistry (IHC). As the same method as the HE staining observation, additionally referring to the diagnostic standards of IHC [30, 31], the expression of mucins protein was evaluated.

2.5. Statistical Analysis. By using SPSS software version 22.0 for Windows, a two-tailed $P<0.05$ was defined as statistical significance in this study. And related data was expressed as the mean \pm standard deviation; one-way ANOVA or $t$-test was applied. For the clinical parameters, Pearson's $\chi^{2}$ test was conducted, and with quantitative variables, Chi-square test with $95 \%$ confidence intervals was screened. 


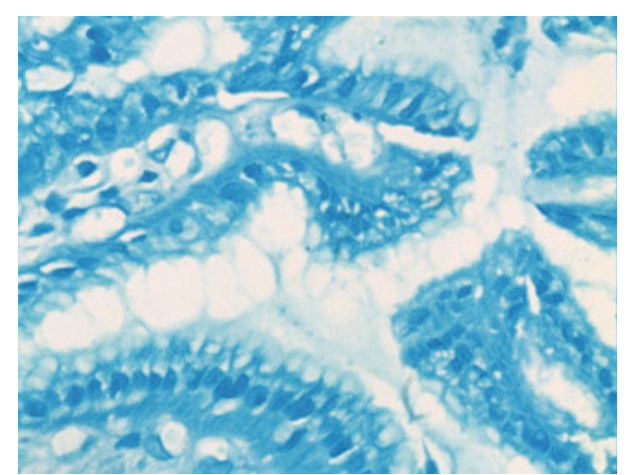

(a)

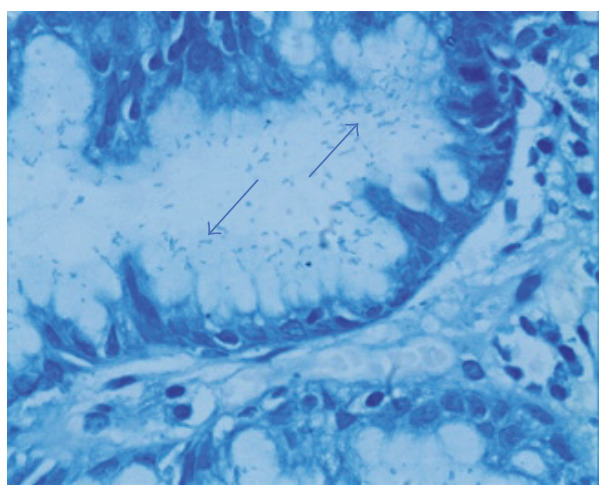

(c)

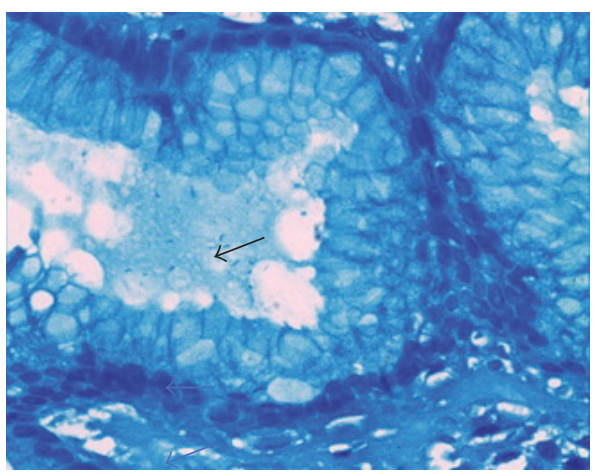

(b)

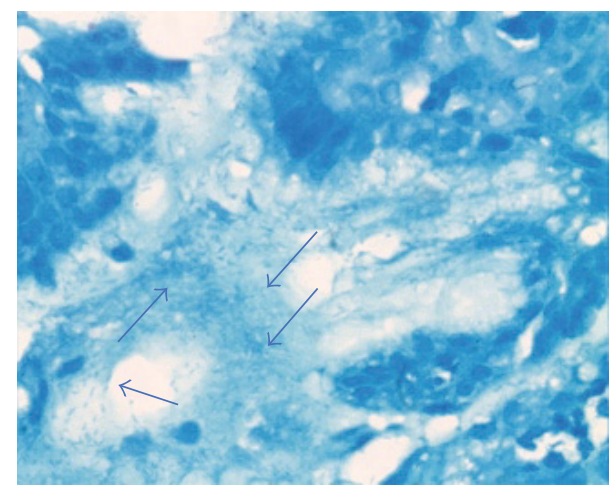

(d)

FIGURE 1: Methylene blue staining (MBS) of gastric mucosa $(\times 400)$ : (a) no Helicobacter pylori (Hp) infection; (b) mild infection: spiral-shaped or small blue rods (arrows) Hp are visible in the gastric pit or mucus layer (arrow); (c) moderate infection; (d) severe infection. The arrow refers to $\mathrm{Hp}$ infection in the gastric mucosa.

\section{Results}

3.1. The Clinical Parameters of the Subjects Included. As shown in Table 1, no statistical significance of different groups exists among gender, diseases, or age, which is the reasonable prerequisite of the following experiment.

3.2. Hp Infection of Gastric Mucosa with MBS. Aiming to increase the sensitivity and specificity for Hp infection test, the specimen was detected with rapid urease; additionally, MBS was performed routinely, and both were defined as positive Hp infection. As shown in Figure 1, under microscope, $\mathrm{Hp}$ is shown like curved or spiral bacillus in the epithelial surface, mucus layer, or gastric pits. According to the different amount of Hp dwelling, none, mild, moderate, and severe infections were screened.

\subsection{Inflammatory Condition of Gastric Mucosa in Different} Groups. With HE staining of specimens, histomorphology appearance of different groups is shown in Figures 2(b)-2(f). In conclusion, compared with $\mathrm{CON}$ and those with negative $\mathrm{Hp}$, the subjects with $\mathrm{Hp}$ infection had more inflammatory cells infiltrating $(P<0.05)$, even with intestinal metaplasia (IM) or dysplasia, regardless of DHSS or SQD. However, no statistical difference was found between DHSS and SQD. By reference to the criteria of IHC [30], the inflammatory degree and activity were present in Figures 2(a) and 2(g).

3.4. Expression of MUC1 and MUC5AC. As shown in Figures 3 and 4, marked by Elivision plus kit, positive expression of MUC1 and MUC5AC was stained with yellow-brown color. And MUC1 was predominantly screened in gastric epithelium mucosa and gland cells, while MUC5AC was mainly detected in the crypt of gastric epithelial gland cells. There was statistical significance for the expression of MUC5AC between the group of DHSS with Hp infection and CON and also between the group of SQD Hp positive and CON $(P<$ 0.05). In contrast, no significance existed for the expression of MUC1.

\section{Discussion}

Nowadays, based on the acknowledgement and communication between CM and Western medicine, some theory has been suggested. For example, Western medicine has been paid more attention to recognize the individual spiritual fulfillment and proposed diagnostic strategies as systembased diagnosis $[17,32]$. On the other hand, for exploration of the spirit of CM, microcosmic point of view of CM has been investigated $[33,34]$. Our team has long been contributing to the research on the essence of deficiency and solid syndrome 


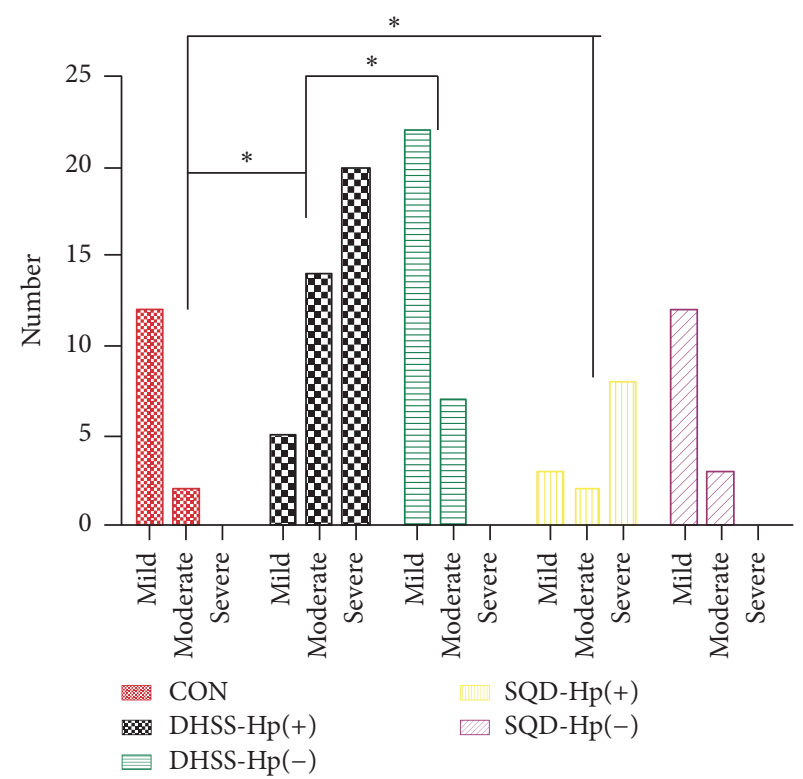

(a)

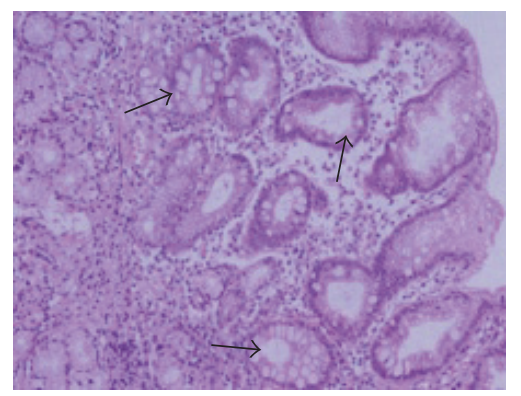

(c)

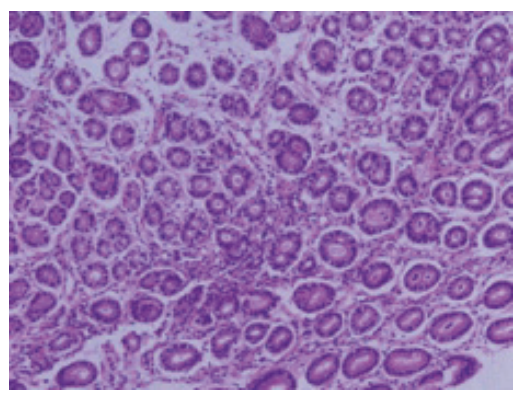

(d)

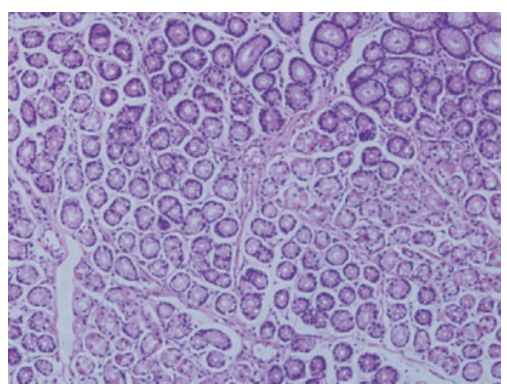

(b)

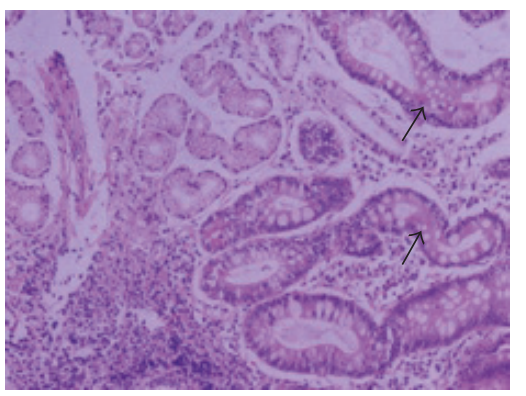

(e)

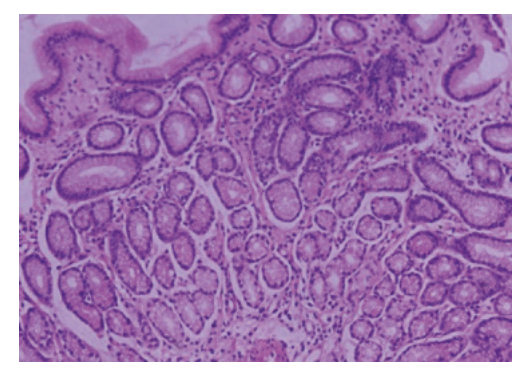

(f)

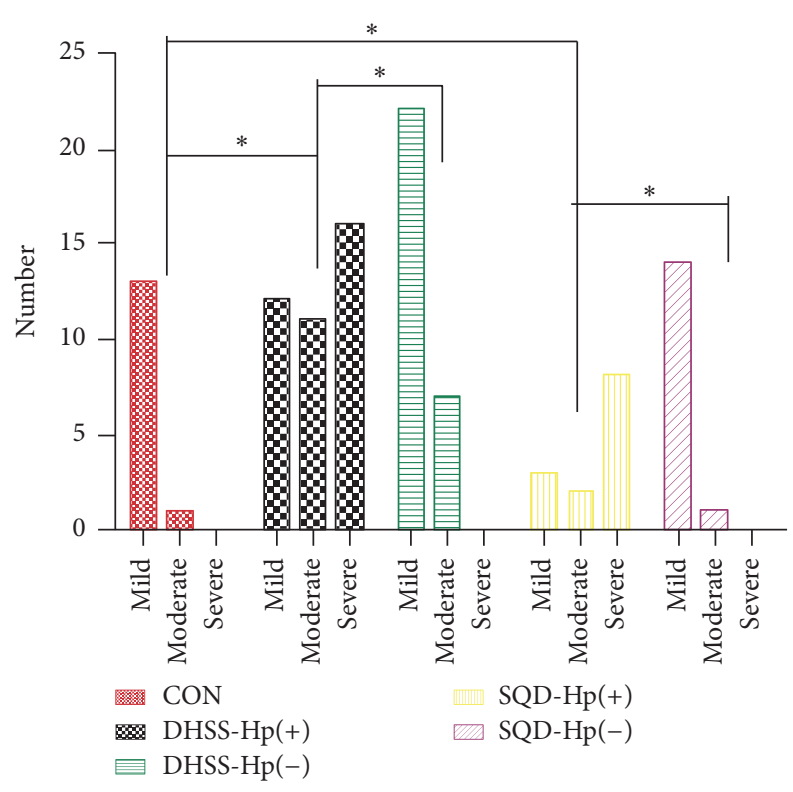

(g)

Figure 2: Histomorphology and inflammatory condition of gastric mucosa samples: (a) inflammatory degree of different groups, ${ }^{*} P<$ 0.05; (b) normal gastric mucosa of control group $(\mathrm{CON})$ : inflammatory cells are rare and gastric glands arrange in order $(\times 100)$; $(c)$ histomorphology of damp-heat syndrome of spleen and stomach (DHSS) with Hp infection: a few number of inflammatory cells infiltrate the gastric mucosa, with intestinal metaplasia visible (IM) (arrow) $(\times 100)$; (d) histomorphology of DHSS without Hp infection: several inflammatory cells and IM or dysplasia present infrequently $(\times 100)$; (e) histomorphology of spleen-qi deficiency syndrome (SQD) with Hp infection ( $\times 100)$; (f) histomorphology of SQD without Hp infection $(\times 100)$; (g) the inflammatory activity of different groups, ${ }^{*} P<0.05$. The arrow refers to Hp infection in the gastric mucosa. 


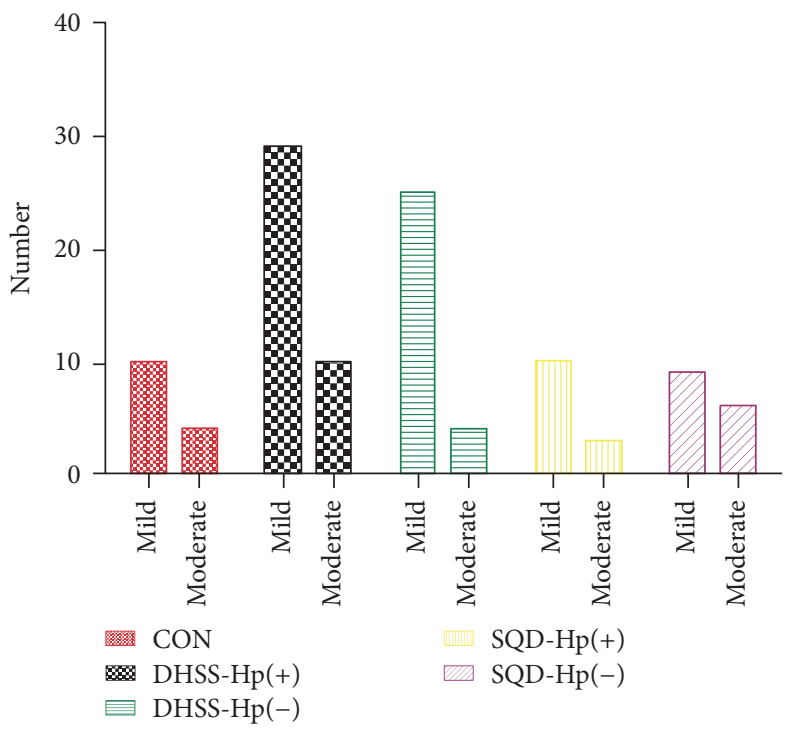

(a)

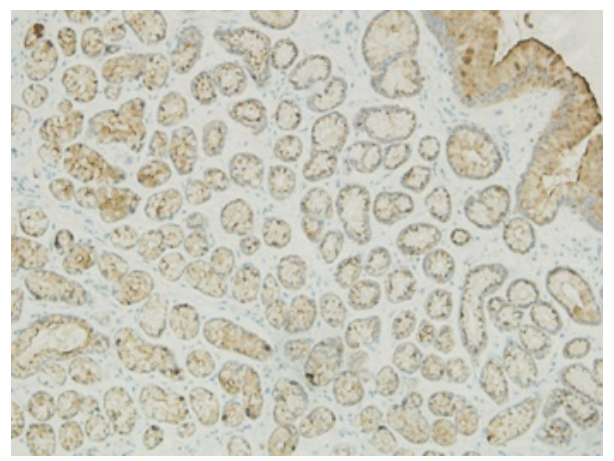

(b)

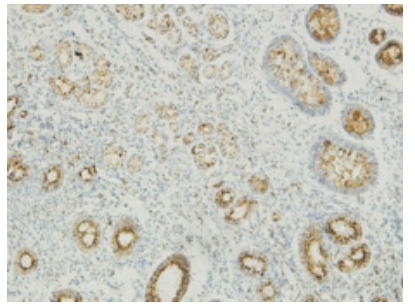

(c)

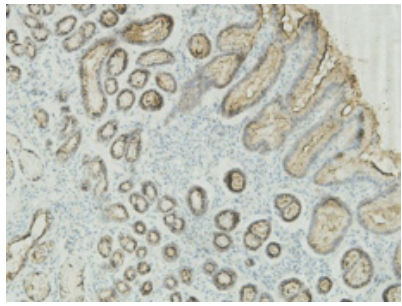

(d)

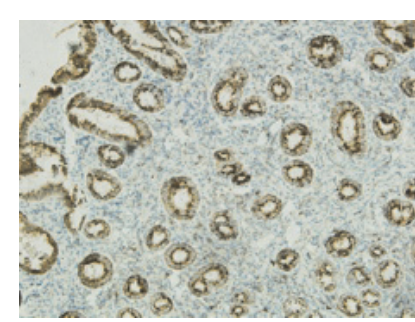

(e)

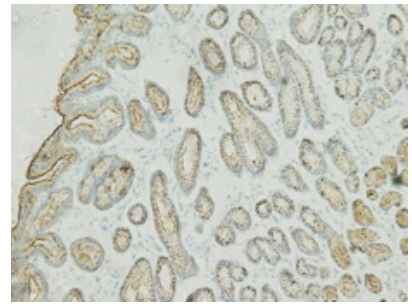

(f)

FIGURE 3: Expression of MUC1: (a) expression of MUC1 in different groups: no statistical difference was observed; (b) expression of MUC1 in control group (CON) ( $\times 100)$; (c) expression of MUC1 in damp-heat syndrome of spleen and stomach (DHSS) with Hp infection ( $\times 100)$; (d) expression of MUC1 in DHSS with Hp negative ( $\times 100)$; (e) expression of MUC1 in spleen-qi deficiency syndrome (SQD) with Hp infection $(\times 100)$; (f) expression of MUC1 in SQD with Hp negative $(\times 100)$.

of spleen and stomach; previous studies on the elucidation of the theory of DHSS and SQD have laid solid foundation for this research $[28,35]$.

As we all know, Hp has been considered as I carcinogen for human beings [36], and continuous infection can cause CG, PU, IM, dysplasia, and even gastric malignancy [3]. In the point view of CM, Hp invasion and colonization in stomach are the representative of evil-qi, while the protection barrier for Hp (gastric epithelium) is the delegate of healthy-qi, whereas mucins (MUCs) are the critical polyprotein components of the gastric epithelium. Consequently, we investigated the relationship of MUCs and the deficiency and solid syndrome of spleen and stomach triggered by Hp infection in order to imply the syndrome spirit of CM.

The human MUC consists of secreted mucins and transmembrane ones, in which MUC1 is transmembrane glycoprotein involved in the signal of epithelial-mesenchymal transition (EMT), while MUC5AC is secreted protein [13], recognized as the major receptor for $\mathrm{Hp}$ in the human stomach [37]. There is evidence that MUC1 is a physical barrier to protect gastric mucosa from Hp dwelling in murine infection model and in vitro experiment; in turn, by injuring the 


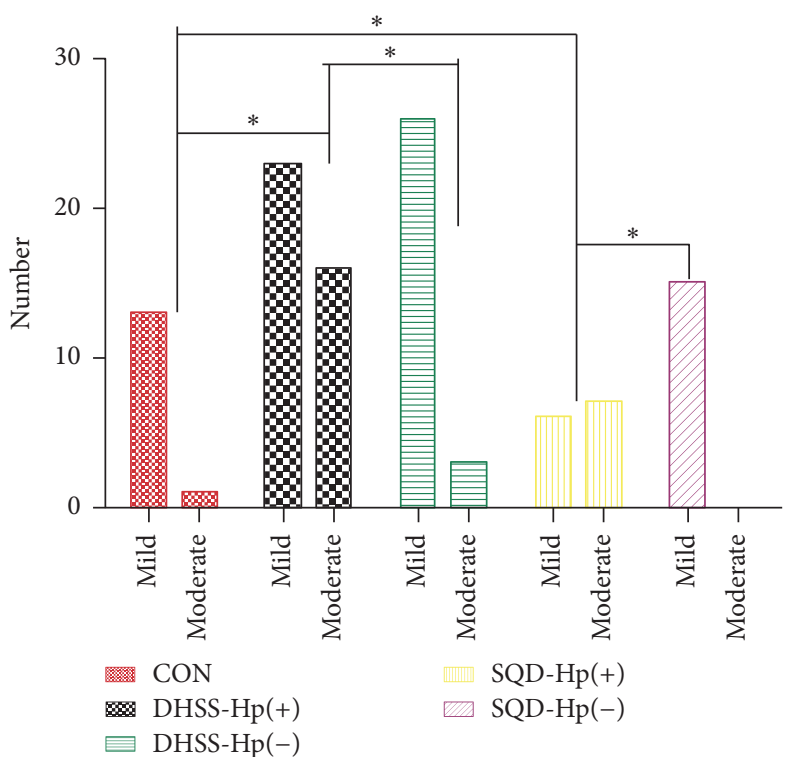

(a)

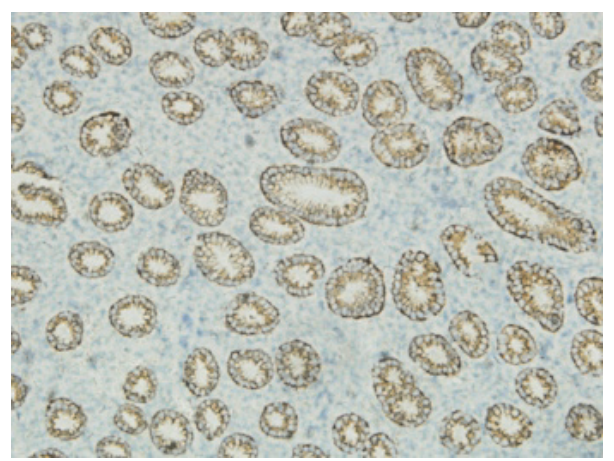

(b)

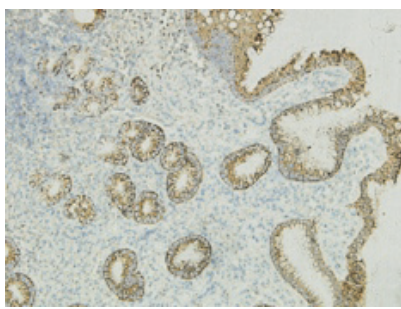

(c)

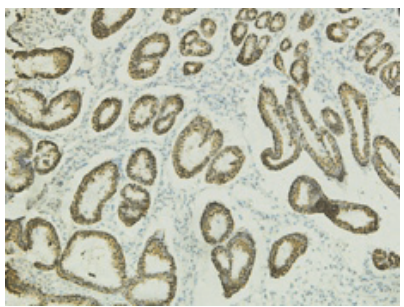

(d)

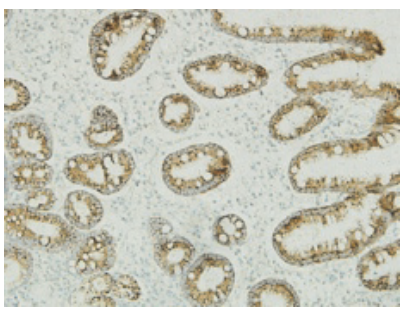

(e)

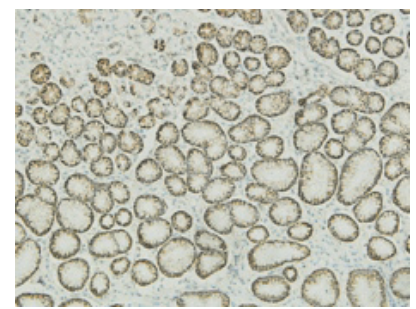

(f)

FigURE 4: Expression of MUC5AC: (a) expression of MUC5AC in different groups, ${ }^{*} \mathrm{P}<0.05$; (b) expression of MUC5AC in control group $(\mathrm{CON})(\times 100)$; (c) expression of MUC5AC in damp-heat syndrome of spleen and stomach (DHSS) with Hp infection $(\times 100)$; (d) expression of MUC5AC in DHSS with Hp negative (×100); (e) expression of MUC5AC in spleen-qi deficiency syndrome (SQD) with Hp infection $(\times 100)$; (f) expression of MUC5AC in SQD with Hp negative $(\times 100)$.

physical barrier, Hp infection would decrease the expression of MUC1 [9-11]. However, for human gastric tissues, studies have shown that no significant association exists between the expression of $\mathrm{MUCl}$ and $\mathrm{Hp}$ infection, which is in accordance with our results in this study, whereas it was overexpressed in the dysplasia and adenocarcinoma tissue, showing its carcinogen characteristics $[12,38]$.

The deficiency and solid syndrome of spleen and stomach is the dynamic procession of transportation and transformation for spleen with the whole body. And the deficiency and solid syndrome is just another form of Yin and Yang like Tai Ji symbol, both of which grasp each other but are also present within each other. Namely, the two sides alter as ecologic succession to sustain dynamic equilibrium, and change of some critical element may disturb the homeostasis. Although, compared with CON, the expression of MUC1 was not different, we still cannot conclude that MUC1 has no association with SQD and DHSS, because Hp inhabitant in gastric epithelium is like wind invading the skin barrier; of course, there is no change for the skin in a modern point of 
view, but it is considered to be due to insecurity of the interstices in CM.

In addition, previous study has shown that only small portion of subjects infected with Hp will develop into malignancy, and the abnormal expression of MUCl acts like oncogene $[12,39]$. Therefore, we cannot deny that MUC1 sustaining may be the related element of dynamic equilibrium regardless of the deficiency and solid syndrome.

On the other hand, for the expression of MUC5AC, results show some difference and even contradiction in different teams. By detection of the expression of endoscopic biopsy with IHC, Kocer et al. [14], have demonstrated that MUC5AC was decreased in patients with $\mathrm{Hp}$ positive, with its localization in the superficial epithelium, upper parts of gastric glands, and dysplastic areas but not in IM. In contrast, with the same method, Park et al. [40], elucidated that MUC5AC was overexpressed in IM of young adults compared to normal and $\mathrm{Hp}$-infected gastric mucosa of children. In consistence with our study, as Figure 4 shows, the extent of MUC5AC expression with Hp infection was more than that with no infection. We speculated that it might result from the fact that the specimens with $\mathrm{Hp}$ positive included in our study showed more IM and dysplasia compared with CON and those with no Hp infection (Figure 2).

In the circumstance of Hp long-time inhabitant in gastric epithelium, to protect the gastric epithelium from deeper damage, MUCs (mainly MUC5AC and MUC1) serve as healthy-qi resistant to $\mathrm{Hp}$. From the holism perspective, the individual syndrome can demonstrate the two sides: deficiency (SQD) and solid (DHSS), but it is another relative homeostasis for gastric epithelium to get dynamic equilibrium showing higher expression of MUC5AC and no change of MUC1. We speculate that it may be the evidence that stomach-qi was still strong even though subjects showed SQD until the equilibrium was broken when showing abnormal expression of MUC1. Consequently, the deficiency and solid syndrome is just relative condition like Tai Ji symbol.

However, with $\mathrm{Hp}$ positive, no statistical significance was found between the groups of DHSS and SQD $(P>0.05)$; we still cannot conclude that MUC5AC gets no correlation with the difference of deficiency and solid syndrome of spleen and stomach because of the limited subjects. Thus, in the following, the extension of included samples is the critical approach. Furthermore, investigation of the single nucleotide polymorphisms (SNPs) for the genotype of MUC1 and MUC5AC may be another method to imply the essence of deficiency and solid syndrome of spleen and stomach.

\section{Disclosure}

Ling $\mathrm{Hu}$ and Wanqun Chen are co-first authors.

\section{Conflicts of Interest}

The authors declare that there are no conflicts of interest with respect to the authorship and/or publication of this paper.

\section{Authors' Contributions}

Ling $\mathrm{Hu}$ and Wanqun Chen contributed equally to this work. Ling $\mathrm{Hu}$ conceived and supervised the study; Ming
Cheng and Ting Zhang performed experiments; Shaoyang Lan, Peiwu Li, and Weijing Chen cooperated the experiments; Wanqun Chen analyzed data; Ling $\mathrm{Hu}$ and Wanqun Chen wrote and revised the manuscript.

\section{Acknowledgments}

This paper was supported by the National Natural Science Foundation of China (nos. 30772689, 81373563, and 81774238 ), the central financial support from the local special funds in colleges and universities (financial education no. [2013] 338), and the program of "South China synergy innovation center of Chinese medicine-gastroenterology and brain disease creative research team" (financial education no. [2014] 488).

\section{References}

[1] S. Suerbaum and P. Michetti, "Helicobacter pylori infection," The New England Journal of Medicine, vol. 347, no. 15, pp. 1175-1186, 2002.

[2] J. Parsonnet, G. D. Friedman, D. P. Vandersteen et al., "Helicobacter pylori infection and the risk of gastric carcinoma," The New England Journal of Medicine, vol. 325, no. 16, pp. 1127-1131, 1991.

[3] E. Yakirevich and M. B. Resnick, "Pathology of gastric cancer and its precursor lesions," Gastroenterology Clinics of North America, vol. 42, no. 2, pp. 261-284, 2013.

[4] D. E. Kirschner and M. J. Blaser, "The dynamics of Helicobacter pylori infection of the human stomach," Journal of Theoretical Biology, vol. 176, no. 2, pp. 281-290, 1995.

[5] M. J. Blaser, "Ecology of Helicobacter pylori in the human stomach," The Journal of Clinical Investigation, vol. 100, no. 4, pp. 759$762,1997$.

[6] C. He, H. Tu, L. Sun et al., "Helicobacter pylori-related host gene polymorphisms associated with susceptibility of gastric carcinogenesis: A two-stage case-control study in Chinese," Carcinogenesis, vol. 34, no. 7, pp. 1450-1457, 2013.

[7] F. Marín, C. Bonet, X. Muñoz et al., "Genetic variation in MUC1, MUC2 and MUC6 genes and evolution of gastric cancer precursor lesions in a long-term follow-up in a high-risk area in Spain," Carcinogenesis, vol. 33, no. 5, pp. 1072-1080, 2012.

[8] M. Rashid, A. S. Teixeira, U. Qureshi, S. P. Pereira, M. R. Novelli, and D. M. Swallow, "Apical MUC1 expression revealed on the foveolar epithelium in $H$. pylori gastritis," British Journal of Cancer, vol. 108, no. 5, pp. 1113-1118, 2013.

[9] M. A. McGuckin, A. L. Every, C. D. Skene et al., "Mucl mucin limits both Helicobacter pylori colonization of the murine gastric mucosa and associated gastritis," Gastroenterology, vol. 133, no. 4, pp. 1210-1218, 2007.

[10] C. Zhang, H. Zhang, L. Yu, and Y. Cao, "Helicobacter pylori Dwelling on the Apical Surface of Gastrointestinal Epithelium Damages the Mucosal Barrier Through Direct Contact," Helicobacter, vol. 19, no. 5, pp. 330-342, 2014.

[11] S. K. Lindén, Y. H. Sheng, A. L. Every et al., "MUC1 limits Helicobacter pylori infection both by steric hindrance and by acting as a releasable decoy," PLoS Pathogens, vol. 5, no. 10, article e1000617, 2009.

[12] D. Boltin, R. Gingold-Belfer, R. Dickman et al., "Gastric mucin expression in first-degree relatives of gastric cancer patients," 
European Journal of Gastroenterology \& Hepatology, vol. 26, no. 7, pp. 710-714, 2014.

[13] D. W. Kufe, "Mucins in cancer: function, prognosis and therapy," Nature Reviews Cancer, vol. 9, no. 12, pp. 874-885, 2009.

[14] B. Kocer, M. Ulas, Y. Ustundag et al., "A confirmatory report for the close interaction of Helicobacter pylori with gastric epithelial MUC5AC expression," Journal of Clinical Gastroenterology, vol. 38, no. 6, pp. 496-502, 2004.

[15] S. E. Baldus, S. P. Mönig, V. Arkenau et al., "Correlation of MUC5AC immunoreactivity with histopathological subtypes and prognosis of gastric carcinoma," Annals of Surgical Oncology, vol. 9, no. 9, pp. 887-893, 2002.

[16] Y. Wang and $\mathrm{A}$. Xu, "Zheng: a systems biology approach to diagnosis and treatments," Science, vol. 346, no. 6216, pp. S13S15, 2014.

[17] J. van der Greef, “Perspective: all systems go," Nature, vol. 480, no. 7378 , article S87, 2011.

[18] G. H. Lv and S. X. Lao, "Establishement and evaluation for the animal model of damp-heat of spleen-stomach syndrome," Journal of Guangzhou University of Traditional Chinese Medicine, no. 03, pp. 231-235, 2005.

[19] F. S. Zhou and L. N. Zhao, "Research on the establishment for the diagnostic standards of spleen-qi deficiency in chronic subepithelial gastritis," Zhong Yi Yao Xue Kan, no. 12, pp. 21782179, 2006.

[20] W. W. Chen, Y. F. Wang, S. X. Lao et al., "Profile of gene differential expression in chronic gatritis with deficiency of spleenqi patients," Zhong Guo Bing Li Sheng Li Za Zhi, no. 01, pp. 148$152,2008$.

[21] J. K. Liang, L. Hu, X. F. Zheng, X. H. Yan, and W. L. Gu, "Study of Th1/Th2 balance on peripheral blood of chronic gastritis patients with pi-wei damp-heat syndrome," Zhong Guo Zhong Xi Yi Jie He Za Zhi, no. 03, pp. 322-324+328, 2012.

[22] P. Malfertheiner, F. K. Chan, and K. E. McColl, "Peptic ulcer disease," The Lancet, vol. 374, no. 9699, pp. 1449-1461, 2009.

[23] J. Y. Fang, W. Z. Liu, Y. Shi, Z. Z. Ge, and S. D. Xiao, "Consensus on chronic gastritis in China - second national consensus meeting on chronic gastritis (14-16 september 2006 Shanghai, China)," Journal of Digestive Diseases, vol. 8, no. 2, pp. 107-119, 2007.

[24] C. Ricci, J. Holton, and D. Vaira, "Diagnosis of Helicobacter pylori: invasive and non-invasive tests," Best Practice \& Research Clinical Gastroenterology, vol. 21, no. 2, pp. 299-313, 2007.

[25] C.-A. Tseng, W.-M. Wang, and D.-C. Wu, "Comparison of the clinical feasibility of three rapid urease tests in the diagnosis of Helicobacter pylori infection," Digestive Diseases and Sciences, vol. 50, no. 3, pp. 449-452, 2005.

[26] F. Bermejo, D. Boixeda, J. P. Gisbert et al., "Rapid urease test utility for Helicobacter pylori infection diagnosis in gastric ulcer disease," Hepatogastroenterology, vol. 49, no. 44, pp. 572-575, 2002.

[27] X. Y. Zheng, Guidelines for the Research of New Clinical Drugs of Chinese Medicine (Trial Implementation), China Medical Science Press, Beijing, China, 2002.

[28] L. Hu, N. J. Cui, Q. Luo, Z. Zhou, and S. X. Lao, "HSP70 and NF- $\kappa$ B mediating damp-heat syndrome of spleen and stomach of chronic gastritis," Journal of Guangzhou University of Traditional Chinese Medicine, no. 06, pp. 587-591+669-670, 2010.

[29] M. F. Dixon, R. M. Genta, J. H. Yardley, and P. Correa, "Classification and grading of gastritis. The updated Sydney System. International Workshop on the Histopathology of Gastritis,
Houston 1994," The American Journal of Surgical Pathology, vol. 20, no. 10, pp. 1161-1181, 1996.

[30] L. Z. Xu and W. T. Yang, "The diagnostic standards for the results of immunohistochemistry," China Oncology, no. 04, pp. 229231, 1996.

[31] T. Zhao, M. G. Zhu, Z. Y. Huang, Y. L. Zhang, S. J. Zhang, and M. F. Li, "Contrastive analysis for the synchronization test of lung cancer gene protein product," Ai Zheng, no. 01, pp. 13-15, 1995.

[32] J. van der Greef, H. van Wietmarschen, J. Schroën, M. Wang, T. Hankemeier, and G. Xu, "Systems biology-based diagnostic principles as pillars of the bridge between Chinese and Western medicine," Planta Medica, vol. 76, no. 17, pp. 2036-2047, 2010.

[33] R. Li, T. Ma, J. Gu, X. Liang, and S. Li, "Imbalanced network biomarkers for traditional Chinese medicine syndrome in gastritis patients," Scientific Reports, vol. 3, article 1543, 2013.

[34] Z. J. Zheng, "Exploration of microcosmic Chinese medicine used by western medicine," Zhongguo Zhong Xi Yi Jie He Za Zhi, vol. 35, no. 2, pp. 133-136, 2015.

[35] L. Hu, G. L. Chen, and W. W. Chen, "The application of spleen deficiency theory: the topic serie of inheritant and application spleen and stomach theory (4)," Journal of Traditional Chinese Medicine, no. 14, pp. 1174-1177, 2012.

[36] "Schistosomes, liver flukes and Helicobacter pylori. IARC Working Group on the Evaluation of Carcinogenic Risks to Humans. Lyon, 7-14 June 1994," IARC Monographs on the Evaluation of Carcinogenic Risks to Humans, vol. 61, pp. 1-241, 1994.

[37] J. H. B. Van de Bovenkamp, J. Mahdavi, A. M. Korteland-Van Male et al., "The MUC5AC glycoprotein is the primary receptor for Helicobacter pylori in the human stomach," Helicobacter, vol. 8, no. 5, pp. 521-532, 2003.

[38] J. B. E. Benjamin, V. Jayanthi, and H. Devaraj, "MUC1 expression and its association with other aetiological factors and localization to mitochondria in preneoplastic and neoplastic gastric tissues," Clinica Chimica Acta, vol. 411, no. 23-24, pp. 2067-2072, 2010.

[39] G. Sachs and D. R. Scott, "Helicobacter pylori: Eradication or preservation," F1000 Medicine Reports, vol. 4, no. 1, article no. 7, 2012.

[40] J. S. Park, J.-S. Yeom, J.-H. Seo et al., "Immunohistochemical Expressions of MUC2, MUC5AC, and MUC6 in Normal, Helicobacter pylori Infected and Metaplastic Gastric Mucosa of Children and Adolescents," Helicobacter, vol. 20, no. 4, pp. 260268, 2015. 


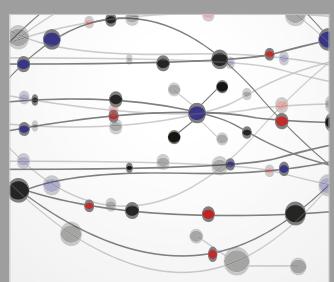

The Scientific World Journal
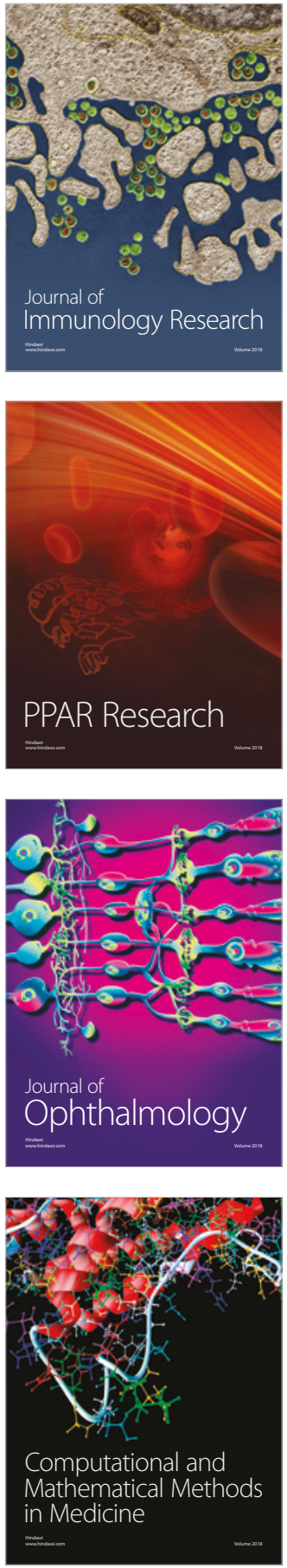

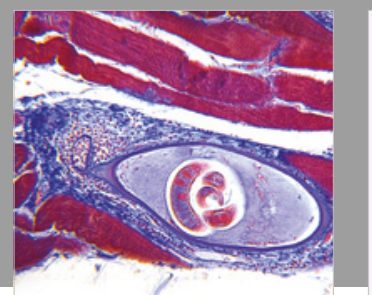

Gastroenterology Research and Practice

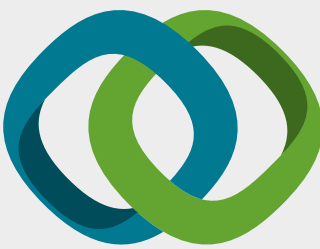

\section{Hindawi}

Submit your manuscripts at

www.hindawi.com
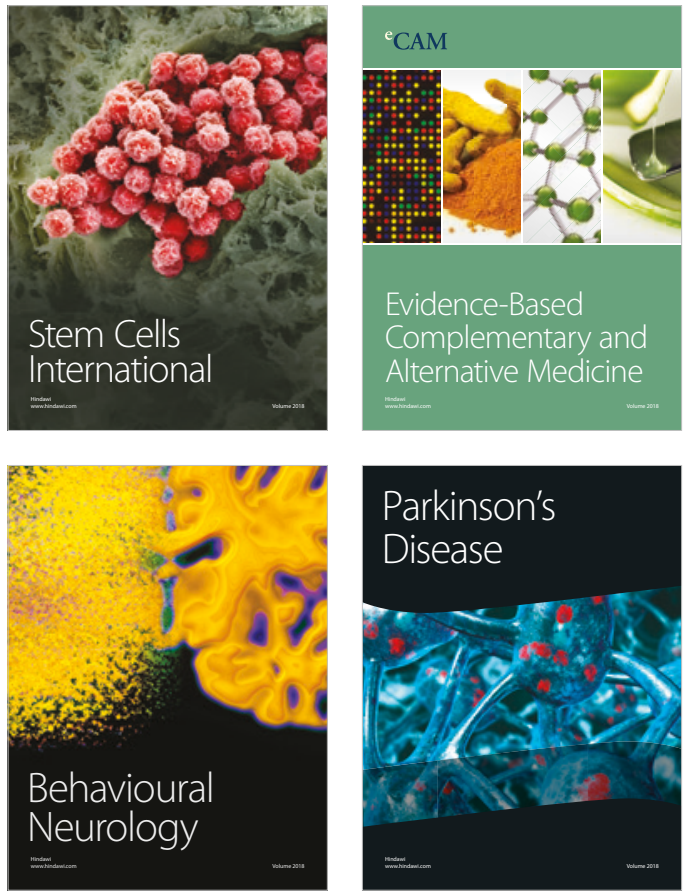

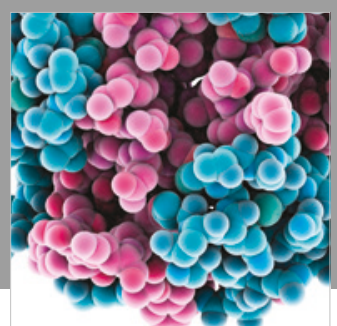

ournal of

Diabetes Research

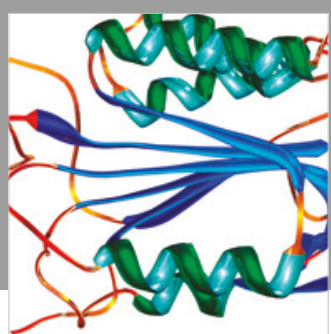

Disease Markers
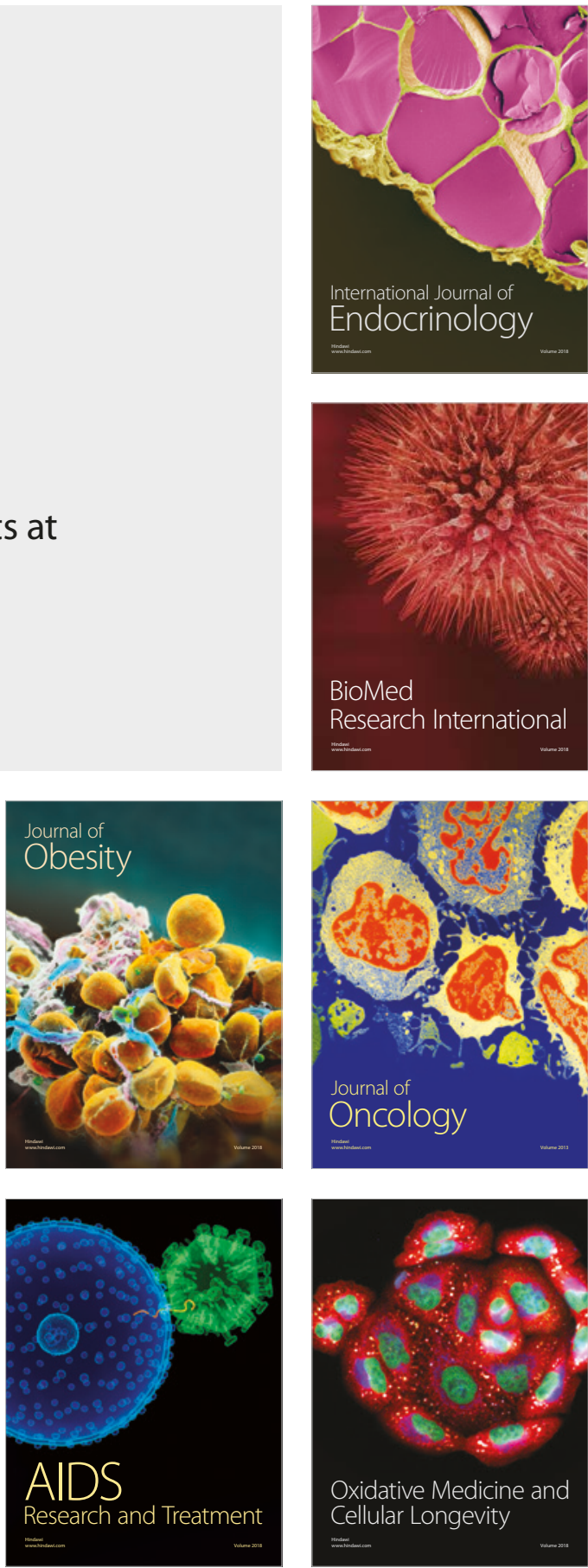